\title{
Comparative nucleic acid transfection efficacy in primary hepatocytes for gene silencing and functional studies
}

\author{
Jae-Seung Park', Sneha Surendran', Lisa M Kamendulis ${ }^{2}$ and Núria Morral ${ }^{1,3^{*}}$
}

\begin{abstract}
Background: Primary hepatocytes are the best resource for in vitro studies directed at understanding hepatic processes at the cellular and molecular levels, necessary for novel drug development to treat highly prevalent diseases such as non-alcoholic steatohepatitis, cardiovascular disease and type 2 diabetes. There is a need to identify simple methods to genetically manipulate primary hepatocytes and conduct functional studies with plasmids, small interfering RNA (siRNA) or microRNA (miRNA). New lipofection reagents are available that have the potential to yield higher levels of transfection with reduced toxicity.

Findings: We have tested several liposome-based transfection reagents used in molecular biology research. We show that transfection efficiency with one of the most recently developed formulations, Metafectene Pro, is high with plasmid DNA ( $>45 \%$ cells) as well as double stranded RNA (>90\% with siRNA or microRNA). In addition, negligible cytotoxicity was present with all of these nucleic acids, even if cells were incubated with the DNA:lipid complex for 16 hours. To provide the proof of concept that these conditions can be used not only for overexpression of a gene of interest, but also in RNA interference applications, we targeted two liver expressed genes, Sterol Regulatory Element-Binding Protein-1 and Fatty Acid Binding Protein 5 using plasmid-mediated short hairpin RNA expression. In addition, similar transfection conditions were used to optimally deliver siRNA and microRNA.

Conclusions: We have identified a lipid-based reagent for primary hepatocyte transfection of nucleic acids currently used in molecular biology laboratories. The conditions described here can be used to expedite a large variety of research applications, from gene function studies to microRNA target identification.
\end{abstract}

\section{Background}

The liver is a critical tissue that controls carbohydrate and lipid homeostasis. Highly prevalent human conditions, including obesity, cardiovascular disease, the metabolic syndrome, non-alcoholic steatohepatitis (NASH), or type 2 diabetes, are characterized by alterations in hepatic glucose and/or lipid metabolism. Deciphering the molecular mechanisms that mediate metabolic responses will open the possibility for the development of more effective therapies.

Establishing the function of genes and/or validating their role in disease is commonly attained through

\footnotetext{
* Correspondence: nmorralc@iupui.edu

'Department of Medical and Molecular Genetics, Indiana University School of Medicine, 975 West Walnut St, IB130, Indianapolis, Indiana 46202, USA Full list of author information is available at the end of the article
}

generation of null alleles. The discovery of RNA interference (RNAi) has provided novel avenues to accelerate gene function studies as well as drug target discovery [1-5]. RNAi is triggered by double stranded RNA (dsRNA) of variable lengths, typically 21 to 28 nucleotides (nt). These can be chemically synthesized molecules (known as small interfering RNA, or siRNA) that upon delivery into cells or tissues, block gene expression for a few days. Re-administration is necessary for a sustained silencing effect. Alternatively, short hairpin RNA (shRNA) can be used to provide a continuous source of silencing molecules. To generate shRNA, expression cassettes are engineered using RNA polymerase II or RNA polymerase III promoters. The shRNA is transcribed in the nucleus as a linear molecule that folds to generate a stem of approximately 21-nt,

\section{C) Biomed Central}


connected with a loop sequence of variable length. The shRNA hairpin structure is cleaved into a siRNA [6-8]. Once an effective silencing shRNA construct is identified, the expression cassette can be incorporated into viral vectors for delivery to tissues in vivo [9].

In recent years it has become clear that microRNA (miRNA) are crucial modulators of biological functions, affecting processes as important as development and cell cycle stage. It has been estimated that more than $30 \%$ of human genes are regulated by miRNA at an average of 200 genes per miRNA [10-13]. MicroRNA exert their action by binding to the 3' UTR of mRNA and inhibiting protein synthesis or inducing mRNA degradation $[14,15]$. Gene target prediction coupled to experimental confirmation is beginning to yield information on the role of miRNA in normal cellular pathways as well as in disease. MicroRNA target identification is typically attained by transfection using chemically synthesized miRNA mimics (acting as mature miRNA that decrease levels of target mRNA and/or protein).

The primary culture of hepatocytes is regarded as the cellular model with highest similarity to liver physiology, and is the preferred approach to perform functional studies. Primary hepatocytes can be easily obtained by enzymatic digestion with collagenase [16-18]. Compared to other methods, transfection with cationic lipids offers the advantage of being a simple method of gene transfer, shortening gene function studies considerably. In recent years a variety of novel lipofection reagents have been developed with the potential to improve nucleic acid delivery in the absence of toxic effects. Optimization experiments in primary cells are highly time-consuming and expensive to perform. Here, we show transfection conditions with DNA and RNA molecules to knockdown two hepatic genes, Sterol Regulatory ElementBinding Protein-1 (SREBP-1) and Fatty Acid Binding Protein 5 (FABP5), using shRNA-expressing plasmids or siRNA. Similar conditions were successfully used to transfect primary hepatocytes with miRNA.

\section{Results and discussion}

\section{Plasmid transfection optimization}

Critical aspects of mouse primary hepatocyte isolation by collagenase digestion are the strain and age of the animal, which affect cell viability, even if using the same collagenase concentration and perfusion time (data not shown). Using the protocol described in the Methods section, we consistently obtained high cell yield and viability (85-90\%). High transfection efficiency can be obtained in cell lines with essentially all commercial transfection reagents, whereas primary cells in general have proven much more difficult to transfect $[19,20]$. Plasmid transfection, in particular, is more challenging, as these are large molecules and more difficult to be taken up by cells. To analyze the potential of novel lipofection methods to improve primary hepatocyte gene delivery, a $3 \mathrm{~kb}$ plasmid expressing green fluorescent protein (GFP) was complexed to four reagents: Metafectene, Metafectene Pro, Lipofectamine 2000, and Targefect-Hepatocyte. These reagents were selected based on information available from the respective company indicating high transfection efficiency in multiple cell lines and/or primary cells (including primary hepatocytes), in addition to low toxicity. To develop an optimal protocol for transfecting plasmid DNA into primary hepatocytes, DNA:lipid ratio, DNA concentration, culture medium and transfection time were considered. As shown in Figure 1A, we found that transfection was most efficient with all reagents when using a DNA:lipid ratio of 1:4. Targefect yielded the highest transfection efficiency $(45.8 \pm 4.2 \%)$, followed by Metafectene Pro $(40.3 \pm 2.6 \%)$, Lipofectamine $2000(37.3 \pm 3.8 \%)$, and Metafectene $(31.5 \pm 2.9 \%)$ (Figure 1A). We then tested the effect of total amount of DNA, by transfecting cells with 1 to $5 \mu \mathrm{g}$, and maintaining the 1:4 (DNA:lipid) ratio. The highest transfection efficiency in the absence

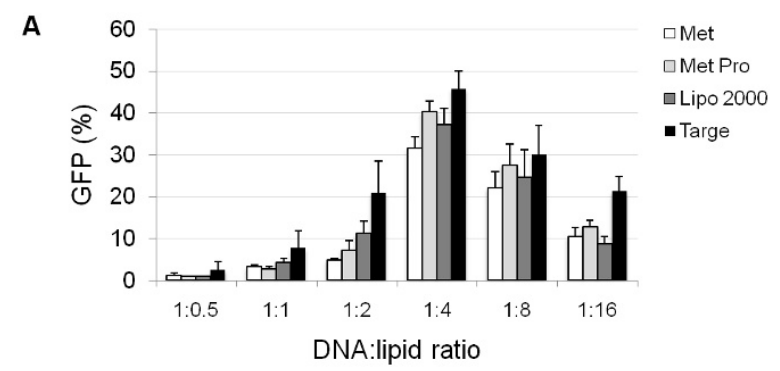

B

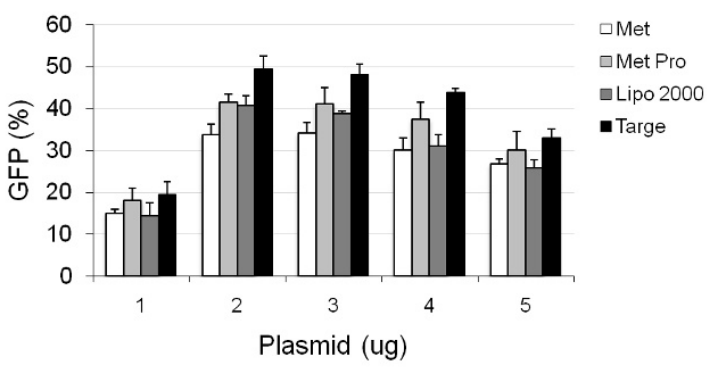

Figure 1 Effect of DNA:lipid ratio and DNA concentration on transfection efficiency. Cells were transfected with pRNAT-H1.1/ neo GFP plasmid complexed with Metafectene (Met), Metafectene Pro (Met Pro), Lipofectamine 2000 (Lipo 2000) or Targefect (Targe), for $16 \mathrm{hr}$. (A) DNA:lipid ratio. Two $\mu \mathrm{g}$ DNA was complexed with lipid at ratios of 1:0.5, 1:1, 1:2, 1:4, 1:8, 1:16. The percentage of GFPpositive cells was determined $24 \mathrm{hr}$ after transfection. Values represent the average of 4 independent experiments. (B) DNA concentration. Using a 1:4 DNA:lipid ratio, cells were transfected with $1,2,3,4$, and $5 \mu \mathrm{g}$ of DNA. The percentage of GFP-positive cells was determined $24 \mathrm{hr}$ after transfection. Values represent the average of 3 independent experiments. 
of toxicity was obtained with $2 \mu \mathrm{g}$ with all reagents (Figure 1B). Because Metafectene gave the lowest transfection efficiency under all conditions, further optimization experiments were conducted without this reagent.

To investigate the influence of medium and transfection time, primary hepatocytes were transfected with Metafecte Pro, Lipofectamine 2000 or Targefect in the presence of DMEM (Figure 2A) or Opti-MEM, with/ without Virofect enhancer, for 3 hours or 16 hours (Figure $2 \mathrm{~A}, 2 \mathrm{~B}, 2 \mathrm{C}$ ). The presence of Opti-MEM resulted in

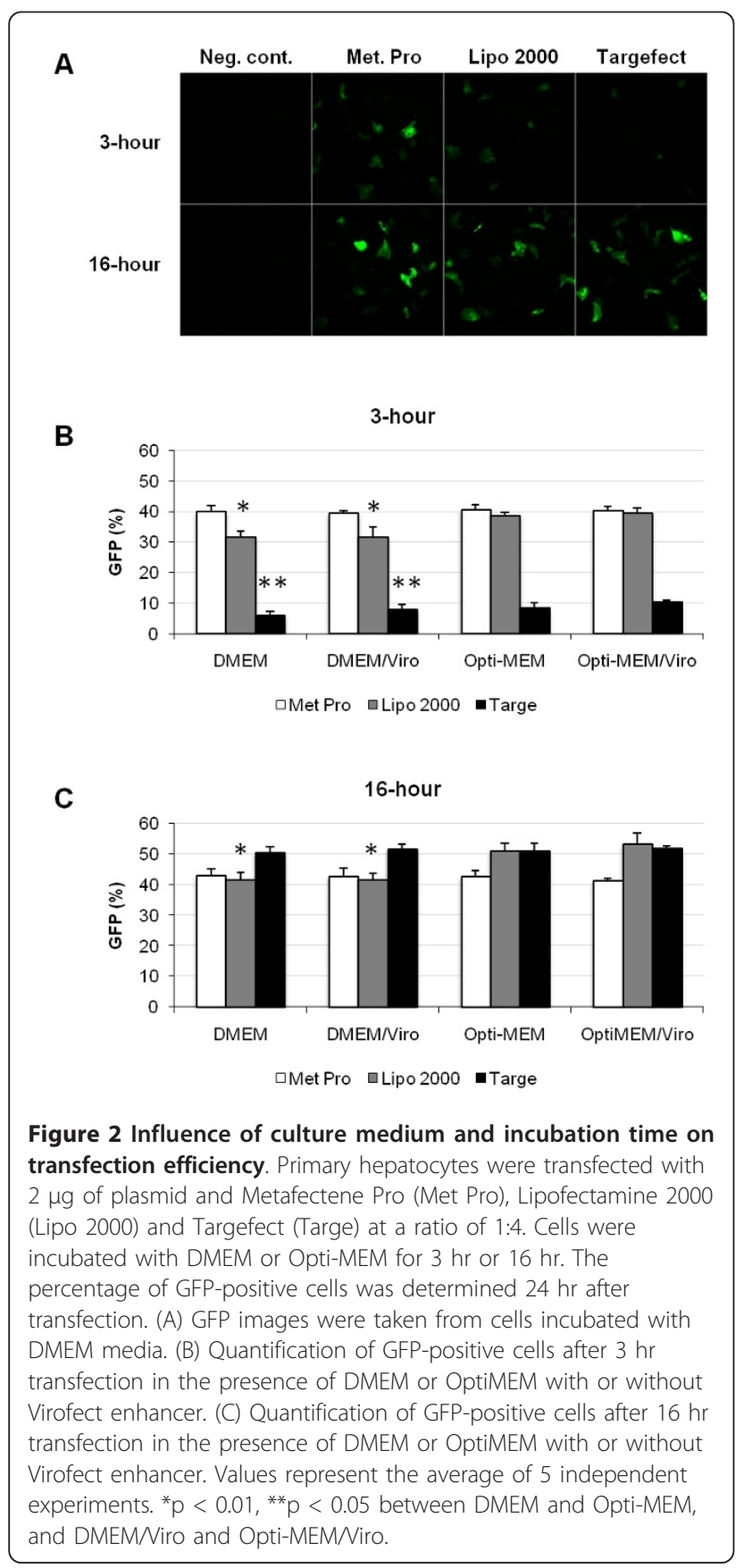

significantly higher transfection rates when Lipofectamine 2000 was used (approximately 10\% increase at both, 3 and 16 hours) (Figure 2B, 2C), a small but significant increase on cells incubated with Targefect for 3 hours (Figure 2B), and no statistically significant difference with Metafectene Pro (Figure 2B, 2C). Virofect enhancer had no significant effect on transfection efficiency when Metafectene Pro, Lipofectamine 2000 or Targefect were used (Figure 2B, 2C). Interestingly, longer transfection time had a large influence on percentage of GFP-positive cells when Targefect was used. For example, in DMEM-incubated cells, $5.8 \pm 1.6 \%$ were GFP-positive when transfection lasted 3 hours, while $50.6 \pm 1.9 \%$ were positive when cells were transfected for 16 hours $(\mathrm{p}<0.01)$. Transfection time also influenced the \% of GFP-positive cells when using Lipofectamine 2000. Approximately a $10 \%$ increase was observed at 16 hours compared to 3 hours $(31.4 \pm 2.3 \%$ at 3 hours and $41.6 \pm 2.5 \%$ at 16 hours with DMEM; p < $0.01 ; 38.6 \pm 1.1 \%$ at 3 hours and $51.2 \pm 2.4 \%$ at 16 hours with Opti-MEM; $\mathrm{p}<0.01)$. Transfection time had a negligible effect with Metafectene Pro $(39.8 \pm 2.2 \%$ at 3 hours and $43.0 \pm 2.1 \%$ at 16 hours, using DMEM; $\mathrm{p}=$ $0.046 ; 40.4 \pm 1.8 \%$ at 3 hours and $42.8 \pm 1.9 \%$ at 16 hours using Opti-MEM; $\mathrm{p}=0.07$ ).

High efficiency of transfection is often associated with toxicity. Ideally, a transfection reagent should yield high transfection efficiency with minimal or no cytotoxicity. To examine this, primary hepatocytes were cultured in the presence of DMEM or Opti-MEM, using Metafectene Pro, Lipofectamine 2000, and Targefect and cytotoxicity was evaluated after 24, 48, and $72 \mathrm{hr}$. As shown in Table 1, there was barely any difference in cytotoxicity among transfection reagents at 24 hours posttransfection; however Metafectene Pro resulted in much less cytotoxicity than Lipofectamine 2000 and Targefect at 48 and 72 hours post-transfection. Targefect yielded the highest level of transfection, but was by far the most

Table 1 Cytotoxic effect induced by transfection reagents

\begin{tabular}{|c|c|c|c|c|}
\hline \multirow[t]{2}{*}{ Reagents } & \multirow[t]{2}{*}{ Media } & \multicolumn{3}{|c|}{ Cytotoxicity } \\
\hline & & $24 \mathrm{hr}$ & $48 \mathrm{hr}$ & $72 \mathrm{hr}$ \\
\hline \multirow[t]{2}{*}{ Negative control } & DMEM & - & - & - \\
\hline & Opti-MEM & - & - & - \\
\hline \multirow[t]{2}{*}{ Metafectene Pro } & DMEM & - & + & + \\
\hline & Opti-MEM & - & + & + \\
\hline \multirow[t]{2}{*}{ Lipofectamine 2000} & DMEM & - & ++ & +++ \\
\hline & Opti-MEM & - & + & ++ \\
\hline \multirow[t]{2}{*}{ Targefect } & DMEM & + & +++ & ++++ \\
\hline & Opti-MEM & - & ++ & ++++ \\
\hline
\end{tabular}

Cytotoxicity was assessed by estimating the percentage of cell density in 6 fields per well, relative to the negative control for each time point. $+:<20 \%$; ++ : $<40 \%$; +++: $<60 \%$; ++++: $<80 \%$ cell death. 
toxic of the three reagents. Lipofectamine 2000 gave slightly higher levels of transfection than Metafectene Pro and similar levels of toxicity when cells were cultured in the presence of Opti-MEM. However, transfection using Lipofectamine 2000 resulted in lower reproducibility than the other reagents, which was affected by elapsed time between cell plating and transfection (data not shown). Overall, Metafectene Pro gave the most consistent results, with least toxicity and shortest transfection time. This reagent has been previously shown to have superior performance in prostate cancer cells and the human embryonic carcinoma (EC) stem cell line NTERA2 [21]. Our data suggest that Metafectene Pro is a suitable reagent for use in primary hepatocytes, either for short-term expression $(<24$ hours $)$ or longer expression ( $>24$ hours).

\section{SREBP-1 and FABP5 silencing in primary hepatocytes}

To provide the proof of concept that the transfection conditions described above are sufficient to silence an endogenous gene, primary hepatocytes were transfected with plasmids expressing shRNA to knock-down the transcription factor SREBP-1 [9] and the fatty acid binding protein FABP5 [22]. As shown in Figure 3, SREBP-1 mRNA expression was significantly reduced to $58 \%$ and $18 \%$ (at 24 and 48 hours, respectively) compared to the levels observed in cells treated with the shSCR control plasmid [expressing a scrambled sequence that does not bind to an mRNA, based on Basic Local Alignment Search Tool (BLAST) analysis (National Center for Biotechnology Information, NIH]. Western blot analysis revealed that SREBP-1 protein levels decreased in parallel to mRNA levels. FABP5 mRNA was reduced to $44 \%$ and $12 \%$ at 24 and 48 hours, respectively, compared to samples treated with shSCR plasmid. FABP5 protein reduction was evident only in samples harvested 48 hours after transfection, suggesting this protein has a long half-life. Thus, when testing the efficacy of customdesigned shRNA sequences, measuring the mRNA of the target gene is a more reliable method to assess the silencing efficacy of the constructs than measuring protein levels. In conclusion, the conditions reported here allow gene silencing of two independent genes by transfection of plasmids expressing shRNA.

When conducting studies to address the function of a gene, using a chemically synthesized siRNA may be the preferred approach. In these experiments as well as in experiments directed at miRNA target identification, it is important to use conditions that result in highest transduction efficiency, to maximize the level of inhibition on mRNA and/or protein expression. To optimize the transfection conditions using a chemically synthesized double stranded RNA molecule, a fluorescently labeled miRNA, was used. Cells were transfected with
$0.5,1$, or $2.5 \mu \mathrm{g}$ of Dy547-labeled miRIDIAN microRNA Mimic Transfection Control. All conditions resulted in the presence of a fluorescent signal in the cytoplasm in a high percentage of cells (>90\%). Using $2.5 \mu \mathrm{g}$ of miRNA the intensity in the cytoplasm was slightly stronger than in cells transfected with $1 \mu \mathrm{g}$, but there were large clumps outside of the cells, suggesting that not all nucleic acid was taken by cells. Experiments were repeated in 6 - $\mathrm{cm}$ dishes using the same dsRNA: lipid ratio and 1.5, 3.0 and $4.5 \mu \mathrm{g}$ miRNA. Similar results were observed to those seen in 6-well plates (Figure 4A). No cytotoxicity was observed in any of these conditions for 48 hours.

To test the feasibility of using these conditions for transfection using siRNA, primary hepatocytes were incubated with siRNA to target FABP5, a non-targeting siRNA or with FABP1 siRNA (another member of the FABP family expressed in liver) (Figure 4B). A significant FABP5 knock-down was observed after 72 and 96 hours with the siRNA specific to FABP5. Thus, similar transfection conditions can be used for delivery of large plasmids, miRNA and siRNA in primary hepatocytes.

In addition to be important for gene function studies, plasmid-mediated expression of shRNA in primary hepatocytes has other applications. In vivo RNAi offers the possibility to overcome many of the limitations presented by knock-out animal models. For example, absence of a gene may be incompatible with development, leading to death in utero. Using viral vector delivery of shRNA-expression cassettes it is possible to induce gene silencing in an adult animal. For the liver, in particular, adenovirus-mediated shRNA expression represents an excellent alternative, as the adenovirus has high tropism for hepatocytes [9]. Using the approach described here, several shRNA-expressing plasmids can be tested for their efficacy at silencing the target gene in primary hepatocytes, prior to transferring the most efficient shRNA expression cassette to an adenoviral vector for in vivo studies.

\section{Conclusion}

This study shows efficient transfection of primary hepatocytes and negligible cytotoxicity using plasmid DNA and dsRNA complexed to Metafectene Pro. These conditions can be applied to (i) functional studies where a gene of interest needs to be silenced, (ii) gene overexpression studies, (iii) testing the efficacy of shRNA constructs to target hepatic genes, and (iv) delivery of small RNA molecules, such as siRNA or miRNA mimics, which resulted in $>90 \%$ transfection efficiency. The primary hepatocyte transfection conditions described here can be used to accelerate gene discovery, drug target validation and miRNA target identification studies, that are urgently needed to treat prevalent human diseases 
A

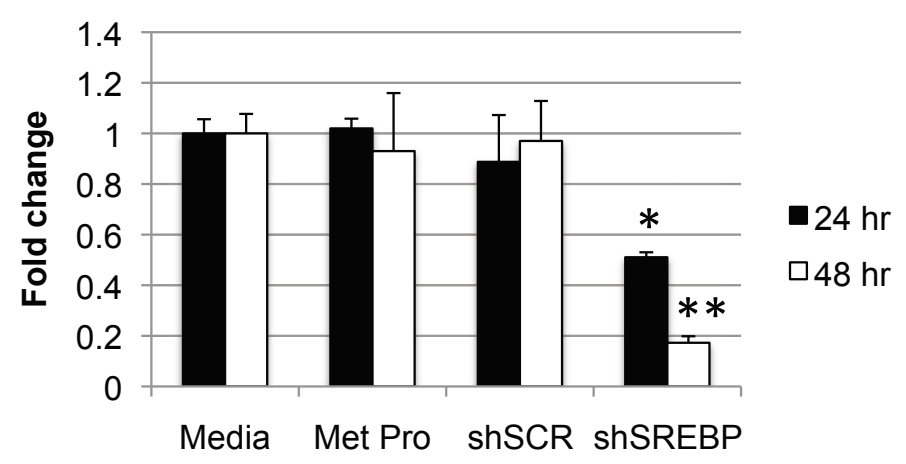

B

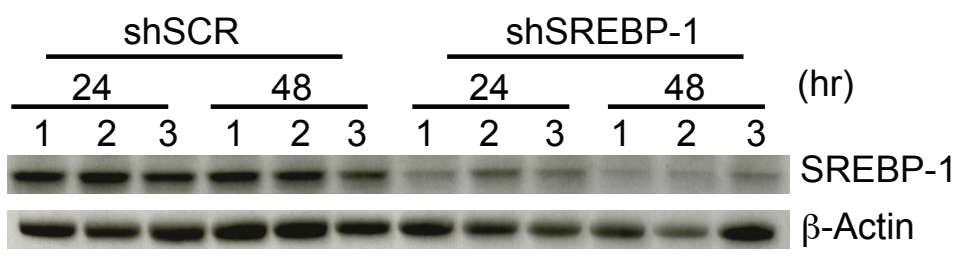

FABP5 mRNA
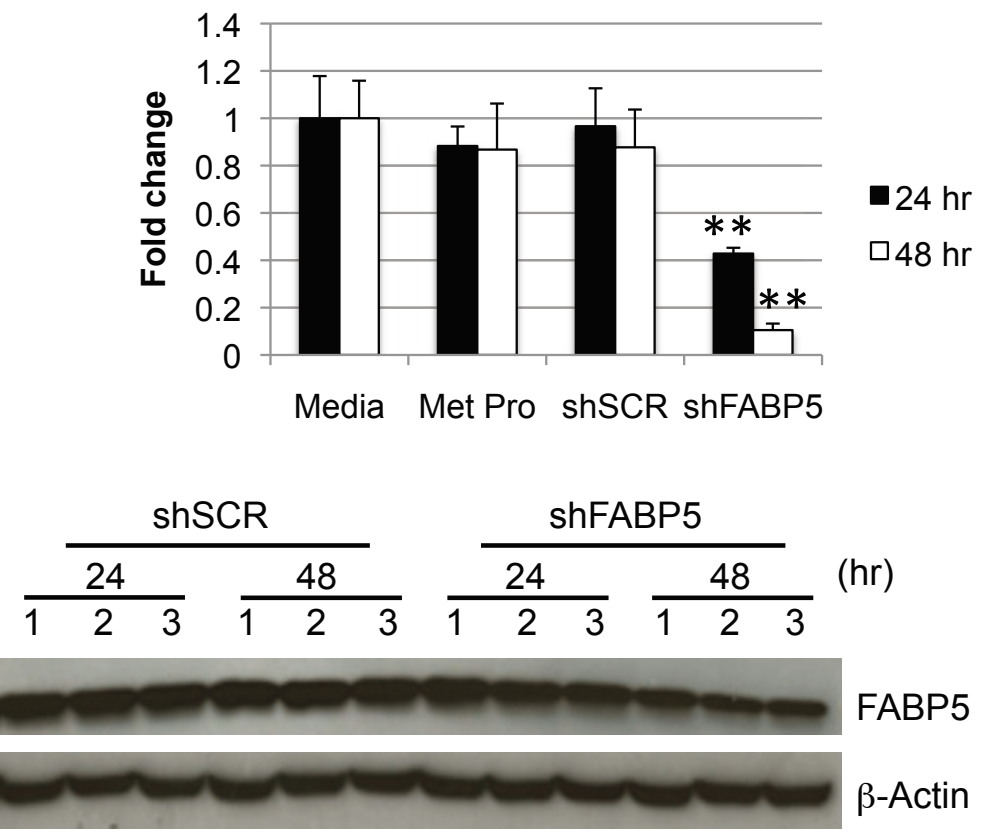

Figure 3 Gene silencing using plasmid transfection. Primary hepatocytes cultured in DMEM were transfected with plasmids $(2 \mu \mathrm{g})$ expressing shRNA to knock-down SREBP1 or FABP5 and Metafectene Pro (1:4 ratio), for 16 hr. (A) SREBP-1 gene silencing; (B) FABP5 gene silencing. The level of mRNA was quantified by real-time RT-PCR ( $A$ and $B$, top). The level of silencing is expressed relative to medium-treated cells. Proteins were quantified by Western blot (A and B, bottom). $\beta$-actin was used as a normalizer. Data are representative of two independent experiments $(n=3) ;{ }^{*} p<0.05 ;{ }^{* *} p<0.01$ shSCR compared to shSREBP-1 or shFABP5. 


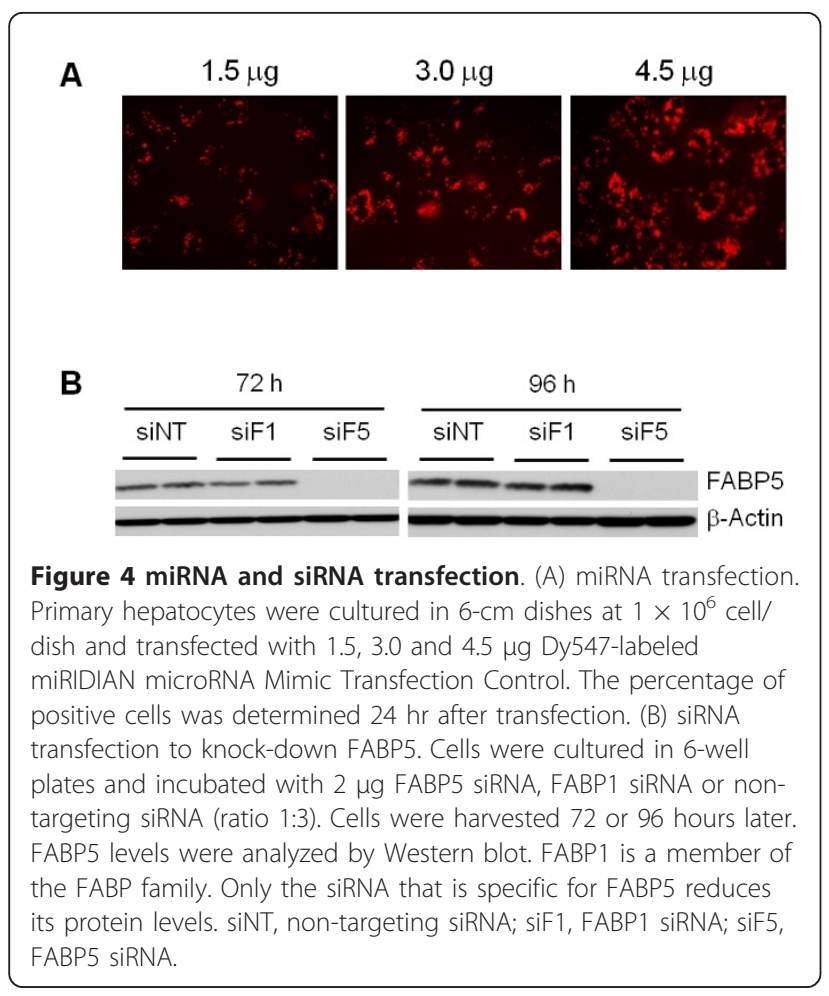

such as obesity, non-alcoholic steatohepatitis, cardiovascular disease, and type 2 diabetes.

\section{Materials and methods Animals}

Male C57BL/6J mice (11 to 12 wks old, 26 to 30 g) were purchased from The Jackson Laboratory (Bar Harbor, ME). Mice were fed rodent chow ad libitum and allowed free access of water. A standard $12 \mathrm{~h}$ light $/ 12 \mathrm{~h}$ dark cycle (7 AM/7 PM) was maintained throughout the experiments. Animal studies were performed in compliance with Indiana University School of Medicine Institutional Animal Care and Use Committee guidelines.

\section{Isolation of mouse primary hepatocytes}

Primary hepatocytes were isolated by collagenase digestion, following a previously described protocol $[16,17]$. Mice were anesthetized by intraperitoneal injection of sodium pentobarbital $(90 \mathrm{mg} / \mathrm{kg})$. The portal vein was cannulated with a 22-gauge intravenous catheter. The liver was perfused with $\mathrm{Ca}^{2+}, \mathrm{Mg}^{2+}$-free Hanks' buffer solution (Invitrogen, Carlsbad, CA) containing $0.5 \mathrm{mM}$ ethylene glycol-bis-(2-aminoethylether) $N, N, N^{\prime}, N^{\prime}$-tetracetic acid (EGTA), and $0.05 \mathrm{M} \mathrm{N}$-2-hydroxyethylpiperazine- $N$-2-ethane sulfonic acid (HEPES) (pH 7.4) maintained at $37^{\circ} \mathrm{C}$, at a rate of $5 \mathrm{ml} / \mathrm{min}$ for $5 \mathrm{~min}$. This was followed by perfusion of the collagenase-containing solution [DMEM (Cellgro, Lawrence, KS) with 0.05\% collagenase Type IA (Sigma, St. Louis, MO)] for
5 minutes $(5 \mathrm{ml} / \mathrm{min})$, with intermittent obstruction of the inferior vena cava for 15 seconds. The liver was transferred to $10 \mathrm{~mm}$ dishes with $15 \mathrm{ml}$ DMEM containing $0.05 \%$ collagenase and was mechanically dissociated into single cells. Ten ml DMEM supplemented with 10\% heat-inactivated fetal bovine serum (FBS) were added to cells to reduce collagenase activity. Cells were filtered through a $70 \mu \mathrm{m}$ pore size strainer (BD, San Jose, CA) and centrifuged at $100 \times \mathrm{g}$ for $5 \mathrm{~min}$ at $10^{\circ} \mathrm{C}$. The cell pellets were gently washed with $20 \mathrm{ml}$ DMEM/10\% FBS, and centrifuged at $100 \times \mathrm{g}$ for $5 \mathrm{~min}$ at $10^{\circ} \mathrm{C}$. This low centrifugation step allows pelleting hepatocytes while significantly reducing the number of other smaller liver cells [17]. Cells were resuspended in DMEM/10\% FBS with antibiotics. Cell viability was assessed by trypan blue dye exclusion (85-90\%). Primary hepatocytes were plated at a density of $4 \times 10^{5}$ cells in $2 \mathrm{ml}$ of DMEM/10\% FBS with antibiotics on 6-well plates, which resulted in $90 \%$ confluency the day after (Corning, Lowell, MA). Medium was replaced 2 hours after plating.

\section{Transient transfection}

After overnight incubation, plated primary hepatocytes were transfected with plasmid pRNAT-H1.1/neo containing a green fluorescent protein (GFP) expression cassette (GeneScript, Piscataway, NJ). Four transfection reagents were tested: Metafectene (Biontex Laboratories $\mathrm{GmbH}$, Munich, Germany), Metafectene Pro (Biontex Laboratories $\mathrm{GmbH}$ ), Lipofectamine 2000 (Invitrogen) and Targefect-Hepatocyte (Targeting Systems, El Cajon, CA). Given that each manufacturer recommends different time frames for incubation of cells with DNA/lipid complexes, 3 and 16 hours were used, as these covered the lowest and highest times suggested. DNA and lipid were diluted in $100 \mu \mathrm{l}$ of medium (in the absence of FBS and antibiotics), in separate tubes. DNA was added to the lipid, gently mixed, and incubated at room temperature for 20 minutes. The complex was added to the cells and the plate was carefully swirled to ensure even distribution. Transfection was conducted in the presence of Dulbecco's modified Eagle's medium (DMEM) or OptiMEM supplemented with 10\% FBS, $100 \mathrm{U}$ of penicillin $\mathrm{G}$ and $100 \mu \mathrm{g}$ of streptomycin per ml. In addition, some experiments (as described in the text) included Virofect in the transfection medium. Cells were incubated at $37^{\circ} \mathrm{C}$ in a $5 \% \mathrm{CO}_{2}$ incubator. Medium was replaced with DMEM or OptiMEM containing 10\% FBS and antibiotics after 3 or 16 hours of transfection. All conditions were tested in duplicate wells and at least in three independent experiments. GFP-positive cells were counted in 6 fields per well on an inverted microscope CKX41 (Olympus, Center Valley, PA) with fluorescence illuminator, BH2-RFL-T3 (Olympus). Fluorescence images were acquired with a Nikon TE 2000 U inverted 
microscope. Images were acquired with a 40x Air EL WD Plan Fluor 0.60NA DIC objective (Melville, NY), a Hamamatsu 1394 Orca-ER Cooled CCD Camera (Bridgewater, NJ). Images were processed with Image J software (NIH, Bethesda, MA).

Cytotoxicity was assessed by estimating the percentage of cell density in 6 fields per well, relative to the negative control for each time point $(+:<20 \%$; ++ : $<40 \%$; $+++:<60 \% ;++++:<80 \%$ cell death).

The construction of shRNA-expressing plasmids to knock-down SREBP-1 and FABP5 has been previously described $[9,22]$. For microRNA transfection optimization in 6-well plates, $0.5,1$, or $2.5 \mu \mathrm{g}$ of Dy547-labeled miRIDIAN microRNA Mimic Transfection Control (Dharmacon, Lafayette, $\mathrm{CO}$ ) was mixed with Metafectene Pro at a 1:3 ratio, following the same protocol described above for plasmids. The experiments were scaled-up to 6 -cm dishes using $1 \times 10^{6}$ cells and 1.5, 3.0 and $4.5 \mu \mathrm{g}$ miRNA. For FABP5 silencing using siRNA, siGenome SMARTpool siRNA (Dharmacon) were used following the conditions described in the text.

\section{qRT-PCR analysis}

Total RNA was isolated using Qiagen (Valencia, CA) RNeasy kit following the manufacturer's protocol. qRTPCR was used to quantify mRNA levels of SREBP-1, FABP5 and $\beta$-actin using primer pairs: SREBP-1, 5'TGCTTTGGAACCTCATCCGC-3' and 5'-AGCAGCAAGATGTCCTCCTGT-3'; FABP5, 5'-CCATGGCCAGCCTTAAGGA-3' and 5'-ACCTTCTCATAGACCCGAGT-3'; $\beta$-actin, 5'-CTACAATGAGCTGCGTGTGGC-3' and 5'ATGGCTGGGGTGTTGAAGGTC-3'. qRT-PCR was performed using an ABI PRISM 7500 instrument (ABI, Foster City, CA) and SYBR Green Qiagen One-Step reverse transcription-PCR kit (Qiagen, Valencia, CA), following the manufacturer's recommendations. Quantification of mRNA levels was done by analyzing 50 ng of total RNA, in duplicate and comparing $C_{t}$ values with those of the standard curve. The $\beta$-actin gene was used as loading control. Fold changes are expressed relative to the shSCRtransfected group.

\section{Western blot}

Transfected primary hepatocytes were lysed in modified RIPA buffer containing protease inhibitors, as previously described [22]. Protein concentration was determined using the BCA kit from Pierce (Rockford, IL). Proteins (20-40 $\mu \mathrm{g})$ were separated in 4-20\% SDS-polyacrylamide Criterion gels (Bio-Rad, Hercules, CA) and transferred to polyvinylidene difluoride (PVDF) membranes (Bio-Rad). Primary antibodies were used at the following concentrations in overnight incubations at $4^{\circ} \mathrm{C}$ : SREBP-1, 1:400; FABP5, 1:500; $\beta$-actin, 1:500). Secondary antibody was added and incubated at room temperature for 1 hour at the following dilutions: HRPconjugated anti-rabbit IgG, 1:3,000; anti-goat IgG, 1:3,000. The anti-SREBP- 1 and $\beta$-actin antibodies were purchased from Santa Cruz Biotechnology (Santa Cruz, CA). The anti-FABP5 and all secondary antibodies were purchased from R\&D Systems (Minneapolis, MN). Blots were developed with Immun-Star (Bio-Rad) and exposed to ECL film (GE Healthcare).

\section{Statistical analysis}

All experimental conditions were done in duplicate and repeated in at least two separate hepatocyte isolations. Data are presented as the arithmetic mean \pm standard deviation. Statistical differences were calculated with a two-tailed, unpaired Student's t-test. Data were considered significant at $\mathrm{p}<0.05$.

\section{Abbreviations}

siRNA: small interfering RNA; shRNA: short hairpin RNA; miRNA: microRNA; RNAi: RNA interference; FABP5: Fatty Acid Binding Protein 5; SREBP-1: Sterol Regulatory Element-Binding Protein-1.

\section{Acknowledgements}

This work was supported by grants from the NIDDK (DK078595 and DK078283), American Diabetes Association (1-08-RA-185), and INGEN (Indiana Genomics Initiative of Indiana University supported in part by Lilly Endowment Inc).

\section{Author details}

${ }^{1}$ Department of Medical and Molecular Genetics, Indiana University School of Medicine, 975 West Walnut St, IB130, Indianapolis, Indiana 46202, USA. ${ }^{2}$ Department of Pharmacology and Toxicology, Indiana University School of Medicine, 635 Barnhill Dr., MS A-401, Indianapolis, Indiana 46202, USA. ${ }^{3}$ Department of Biochemistry and Molecular Biology, Indiana University School of Medicine, 635 Barnhill Dr., MS 4053, Indianapolis, Indiana 46202, USA.

\section{Authors' contributions}

JSP - conducted transfection and molecular biology experiments with plasmids and siRNA, and wrote parts of the manuscript; SS - conducted transfection experiments with miRNA; LMK - assisted in setting up the primary hepatocyte isolation protocol; NM - designed, supervised and coordinated experiments, as well as wrote the paper. All authors have read and approved the manuscript.

\section{Competing interests}

The authors declare that they have no competing interests.

Received: 18 May 2010 Accepted: 18 January 2011

Published: 18 January 2011

\section{References}

1. Romano N, Macino G: Quelling: transient inactivation of gene expression in Neurospora crassa by transformation with homologous sequences. Molecular microbiology 1992, 6(22):3343-3353.

2. Pal-Bhadra M, Bhadra U, Birchler JA: Cosuppression in Drosophila: gene silencing of Alcohol dehydrogenase by white-Adh transgenes is Polycomb dependent. Cell 1997, 90(3):479-490.

3. Elbashir SM, Harborth J, Lendeckel W, Yalcin A, Weber K, Tuschl T: Duplexes of 21-nucleotide RNAs mediate RNA interference in cultured mammalian cells. Nature 2001, 411(6836):494-498.

4. Hannon GJ: RNA interference. Nature 2002, 418(6894):244-251.

5. Tang G, Reinhart BJ, Bartel DP, Zamore PD: A biochemical framework for RNA silencing in plants. Genes Dev 2003, 17(1):49-63. 
6. Gregory RI, Chendrimada TP, Cooch N, Shiekhattar R: Human RISC couples microRNA biogenesis and posttranscriptional gene silencing. Cell 2005, 123(4):631-640.

7. Matranga C, Tomari Y, Shin C, Bartel DP, Zamore PD: Passenger-strand cleavage facilitates assembly of siRNA into Ago2-containing RNAi enzyme complexes. Cell 2005, 123(4):607-620.

8. Rand TA, Petersen S, Du F, Wang X: Argonaute2 cleaves the anti-guide strand of siRNA during RISC activation. Cell 2005, 123(4):621-629.

9. Ruiz R, Witting $S R$, Saxena R, Morral N: Robust hepatic gene silencing for functional studies using helper-dependent adenovirus vectors. Hum Gene Ther 2009, 20:87-94.

10. Lewis $B P$, Burge $C B$, Bartel DP: Conserved seed pairing, often flanked by adenosines, indicates that thousands of human genes are microRNA targets. Cell 2005, 120(1):15-20.

11. Sood P, Krek A, Zavolan M, Macino G, Rajewsky N: Cell-type-specific signatures of microRNAs on target mRNA expression. Proc Natl Acad Sci USA 2006, 103(8):2746-2751.

12. Filipowicz W, Bhattacharyya SN, Sonenberg N: Mechanisms of posttranscriptional regulation by microRNAs: are the answers in sight? Nature reviews 2008, 9(2):102-114.

13. Barbato C, Ruberti F, Cogoni C: Searching for MIND: microRNAs in neurodegenerative diseases. J Biomed Biotechnol 2009, 2009:871313.

14. Liu J, Valencia-Sanchez MA, Hannon GJ, Parker R: MicroRNA-dependent localization of targeted mRNAs to mammalian P-bodies. Nat Cell Biol 2005, 7(7):719-723.

15. Pillai RS, Bhattacharyya SN, Filipowicz W: Repression of protein synthesis by miRNAs: how many mechanisms? Trends Cell Biol 2007, 17(3):118-126.

16. Klaunig JE, Goldblatt PJ, Hinton DE, Lipsky MM, Trump BF: Mouse liver cell culture. II. Primary culture. In Vitro 1981, 17(10):926-934.

17. Klaunig JE, Goldblatt PJ, Hinton DE, Lipsky MM, Chacko J, Trump BF: Mouse liver cell culture. I. Hepatocyte isolation. In Vitro 1981, 17(10):913-925.

18. Alpini G, Phillips JO, Vroman B, LaRusso NF: Recent advances in the isolation of liver cells. In Hepatology. Volume 20. Baltimore, Md; 1994:(2):494-514

19. Kohrmann M, Haubensak W, Hemraj I, Kaether C, Lessmann VJ, Kiebler MA: Fast, convenient, and effective method to transiently transfect primary hippocampal neurons. J Neurosci Res 1999, 58(6):831-835.

20. Gardmo C, Kotokorpi P, Helander H, Mode A: Transfection of adult primary rat hepatocytes in culture. Biochem Pharmacol 2005, 69(12):1805-1813.

21. Iczkowski KA, Omara-Opyene AL, Klosel R: Metafectene is superior to lipofectamine in the transfection of $\mathrm{G}(\mathrm{s})$ alpha prostate cancer cells. Mol Biotechnol 2004, 28(2):97-103.

22. Witting SR, Brown M, Saxena R, Nabinger S, Morral N: Helper-dependent Adenovirus-mediated Short Hairpin RNA Expression in the Liver Activates the Interferon Response. J Biol Chem 2008, 283(4):2120-2128.

doi:10.1186/1756-0500-4-8

Cite this article as: Park et al:: Comparative nucleic acid transfection efficacy in primary hepatocytes for gene silencing and functional studies. BMC Research Notes 2011 4:8.

\section{Submit your next manuscript to BioMed Central and take full advantage of:}

- Convenient online submission

- Thorough peer review

- No space constraints or color figure charges

- Immediate publication on acceptance

- Inclusion in PubMed, CAS, Scopus and Google Scholar

- Research which is freely available for redistribution 